\title{
ACUTE EFFECT OF WHOLE-BODY VIBRATION COMBINED WITH STATIC STRETCHING ON FEMALE GYMNASTS' LEG AND VERTICAL STIFFNESS DURING RUNNING
}

\author{
Ntallas Constantinos ${ }^{i}$, \\ Dallas George \\ National and Kapodistrian University of Athens, \\ School of Physical Education and Sport Science, \\ Greece
}

\begin{abstract}
:
Although whole-body vibration (WBV) combined with static stretching (SS) have proven a suitable method to enhance flexibility without losing muscular strength, it is still unknown what its effect are on running mechanics. Therefore, the purpose of the study was to examine the effect of a WBV combined with SS (WBVSS) warm up procedure on lower limb stiffness and spatiotemporal variables during running. Twenty-two female

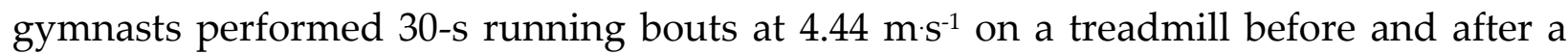
WBVSS intervention stimulus. The WBVSS stimulus included two sets of 20s of SS of four different muscle groups on the vibration platform. Leg and vertical stiffness values were calculated using a spring mass model. The results showed a statistically significant interaction effect on vertical stiffness $\left(\mathrm{K}_{\mathrm{ver}}\right)$, leg stiffness $\left(\mathrm{K}_{\mathrm{leg}}\right)$, change in leg length $(\Delta \mathrm{L})$, maximal ground reaction force (Fmax), contact time $\left(\mathrm{T}_{\mathrm{c}}\right)$, and flight time $\left(\mathrm{T}_{\mathrm{f}}\right)$. Results indicated that, a warm-up including SS combined with WBV did not produce significant effect on mean values of $K_{\text {leg }}$ and $K_{\text {vert, }}$ and related kinetic and kinematic variables.
\end{abstract}

Keywords: stiffness, kinematics, whole-body vibration, gymnasts

\section{Introduction}

The musculotendinous unit (MTU) when exposed to a stretching force result in changes to muscle lengths during its application time, and the muscles return to their original lengths when the force is removed. For the reason that the change in the length of the muscle is not permanent it can be regarded as elastic strain. The quantitative measurement of elastic properties of MTU is their stiffness which defined as the resistance of MTU to deformation. Previous findings support that greater stiffness can be beneficial for movements like hopping and running (Chelly \& Denis, 2002) and that

i Correspondence: email gdallas@phed.uoa.gr 
stiffness has a major influence on rate of force development (RFD), elastic energy storage and sprint kinematics. During these actions the musculoskeletal structures of the legs alternately store and return elastic energy, so the legs could be described as spring based - on spring-mass model theory (Blickhan, 1989; Dalleau et al, 1998; McMahon \& Cheng, 1990). The stiffness of this model is determined by the changes resulting vertical ground reaction force and vertical centre of mass (CoM) displacement. Previous findings refer that stiffness describes the relationship between the force applied to an object (ie: muscles, tendons, fascia, etc.) and the magnitude of the deformation of that object (Brughelli \& Cronin, 2008; Butler et al, 2003) with the most common types of stiffness measured to be leg stiffness $\left(\mathrm{K}_{\mathrm{leg}}\right)$ and vertical stiffness $\left(\mathrm{K}_{\mathrm{vert}}\right)$. Leg and vertical stiffness values were calculated using the spring mass model. An optimal level of stiffness is required to maximize jumping performance (Arampatzis et al, 2001) and high level of $\mathrm{K}_{\text {vert }}$ is related with increased running velocity (Farley et al, 1993). Athletes adjust their level of stiffness depending on the task and surface. During running $\mathrm{K}_{\text {leg }}$ was related with increased stride frequency (Hobara et al, 2010), increased running velocity (Brughelli \& Cronin; Hobara et al, 2010), and decreased stride length. (Derrick et al, 2000). Both types of stiffness can be affected by the functional properties of the MTU, which in turn may be affected by stretching. Various factor acts in a different way on running mechanics; fatigue has a negative influence (Kyröläinen et al, 2000) whereas warm-up affect positively. Previous findings by Pappas et al. (2017) reported that SS and DS although did not affect leg or vertical stiffness; DS affect positively vertical ground reaction force, which resulted in significant increase in flight time, step length and vertical displacement of the CoM and a decrease in step rate.

Athletes incorporated different types of stretching (static, dynamic, PNF) during warm-up in order to reduce the risk of injury during subsequent performance (Nakamura et al, 2014) or to increase the range of motion (ROM) (Dallas et al, 2014; Paradisis et al, 2014). However, other studies support that stretching, particularly slow and static stretching (SS) has a detrimental effect on maximum muscular strength. (Stone et al, 2006). WBV is considered an alternative and suitable neuromuscular training method to enhance muscular function. When a person stands on a vibration platform the oscillations induced by the platform might dampen by the MTU (Abercrombly et al, 2007) which act as a spring-like element during rapid eccentric-concentric actions (Cochrane et al, 2009). This mechanism has been proposed to be related to the stiffness modulation of the stimulated muscle groups during WBV (Cardinale \& Bosco, 2003; Wakeling, Nigg, \& Rozitis, 2002). Furthermore, a great number of studies that have examined the effect of SS in combination with whole body vibration (WBVSS) report a beneficial effect on flexibility (Dallas et al, 2012; 2014; Dallas and Kirialanis, 2013; Kinser et al, 2008; Sands et al, 2008) without impairment of jump performance (Dallas \& Kirialanis, 2013; Kinser et al, 2008; Ronnestad, 2004; Yapicioglu et al, 2013) or found a negative effect (Herda et al, 2009). Specifically, Dallas and Kirialanis. (2013) examined the effect of two different whole-bode Vibration (WBV) intervention protocols (WBV and WBV combined with static stretching: WBVSS) on flexibility and jumping performance on twelve high level 
male artistic gymnasts and found that the percentage improvement of WBV was greater in SJ and CMJ variables compared to WBVSS protocol. In another study, Dallas et al. (2014) examined the acute effects of 3 different warm up methods of stretching (static, proprioceptive neuromuscular facilitation, and stretching exercises on a vibration platform) on flexibility and leg power - jumping performance in eighteen competitive artistic gymnasts finding statistically significant differences for flexibility after all stretching conditions.

A limited number of studies have examined the effect of WBV on leg stiffness (Colson \& Petit, 2013; Cronin et al, 2004; Paradisis et al, 2019; Siu et al, 2010). However, to the best of our knowledge there are no studies that examined the effect of SS combined with WBV (WBVSS) on leg stiffness. Therefore, the purpose of the present study was to investigate the acute effects of WBVSS on leg and vertical stiffness and other spatiotemporal variables during running at submaximal speed. It was hypothesized that 20sec duration of WBVSS could increase leg stiffness through changes in spatiotemporal variables during running, and that WBVSS will be superior to a control condition (SHAM).

\section{Materials and Methods}

\subsection{Participants}

Twenty-two female physical education students coming from various types of gymnastics (artistic gymnastics, rhythmic, aerobic) ([Mean \pm SD] age: $22.59 \pm 3.93$ years, height: $164.09 \pm 4.77 \mathrm{~cm}$, body mass: $58.04 \pm 7.14 \mathrm{~kg}$, training experience: $9.24 \pm 2.36$ years) volunteered to participate in the present study. All participants were healthy with no any injury in the lower limbs in the last 3 months, and recreationally training 12 hours per week in accordance with the academic duties of their faculty. Prior to the testing procedure for each participant, having been informed in detail about the purpose of the study, signed an informed consent form. Ethical approval was gained from the Research Ethics Committee of the National and Kapodistrian University of Athens, School of Physical Education and Sports Science. All participants had previous experience in treadmill running.

\subsection{Design and Procedures}

A randomized, counterbalanced, within-subjects experimental design was conducted to investigate the acute effects of $20 \mathrm{sec}$ of whole-body vibration combined with static stretching (WBVSS) on leg and vertical stiffness during treadmill running. The study carried out over the course of 2 visits on nonconsecutive days (with a 72-h interval) and at the same time of the day. A week before to the commencement of the intervention protocols three familiarization sessions were applied to practice on the treadmill and WBV platform. Before each pre-test, participants completed a 5-minute warm-up on the treadmill at $2.22 \mathrm{~m} \mathrm{~s}^{-1}$ before performing intervention protocol following the post-test measurements immediately after the intervention. 


\subsection{Intervention}

The SS protocol was performed on a WBV platform (Power Plate ${ }^{\circledR}$, Northbrook, USA). The vibration frequency and amplitude were $30 \mathrm{~Hz}$ and $2 \mathrm{~mm}$, respectively. This protocol included 2 set of $20 \mathrm{sec}$ SS on the vibration platform. Participants performed four different exercises to stretch for $20 \mathrm{sec}$ to the point of mild discomfort: a) the knee flexors, b) knee extensors, c) hip extensors, and d) plantar flexors muscles. All participants completed the $1^{\text {st }}$ set of the four muscle groups and then performed the $2^{\text {nd }}$ set. Between each muscle group a 10-second break was applied. The participants in the SHAM protocol remained inactive for the same amount of time.

\subsection{Measurements}

During the pretests and posttests, they performed 10-second running bouts at $4.44 \mathrm{~m} \mathrm{~s}^{-1}$ on a motorized treadmill at their preferred step rate and length. This submaximal speed was chosen as an average of the range of running speeds $\left(3.33-6.67 \mathrm{~m} \mathrm{~s}^{-1}\right)$ used in previous studies (Morin et al., 2005; Pappas et al., 2017). A high-speed video camera (Casio EX-F1; Tokyo, Japan) was placed $1 \mathrm{~m}$ behind the treadmill; perpendicular to the frontal plane, at a height of $40 \mathrm{~cm}$ (Ogueta-Alday et al, 2013) to record participants' strides in a limited area of shoe-treadmill contact during sub-maximal running. Frame rate was selected at $300 \mathrm{~Hz}$, and shutter speed at 1/1250. The Quintic Biomechanics v21 (Sutton, United Kingdom) software was used for the analysis of all video-recorded steps. To calculate vertical and leg stiffness the method described by Morin et al. (2005) was applied. To calculate leg and vertical stiffness the mean values of flight time and contact time of the 10 consecutive steps were used, whereas the estimation of step rate (SR) and step length (SL) the method by Paradisis and Cooke (2001) was applied.

$$
\begin{gathered}
K_{\text {vert }}=F_{\text {max }} \cdot \Delta y_{c}^{-1} \\
F_{\text {max }}=m g \frac{\pi}{2}\left(\frac{t_{f}}{t_{c}}+1\right) \\
\Delta y_{c}=-\frac{F_{\text {max }} t_{c}{ }^{2}}{m \pi^{2}}+g \frac{t_{c}{ }^{2}}{8} \\
K_{\text {leg }}=F_{\text {max }} \cdot \Delta L^{-1} \\
\Delta L=L-\sqrt{L^{2}-\left(\frac{v t_{c}}{2}\right)^{2}+\Delta y_{c}}
\end{gathered}
$$

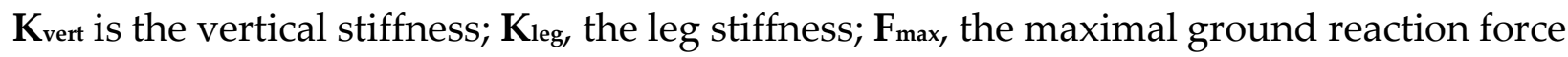
during contact; $\Delta_{\mathbf{y}}$, the vertical displacement of the center of mass; $\mathbf{m}$, the body mass; $\mathbf{t}_{\mathbf{f}}$, the flight time; $t_{c}$, the contact time; $\Delta \mathrm{L}$, the lower limb length variation; and $\mathrm{L}$, the resting lower limb length. 


\subsection{Statistical Analysis}

The IBM SPSS (version 24) was used for statistical analyses. The arithmetic mean, SD, and range were calculated for each variable and trial. Raw data were checked for normality using a Shapiro-Wilk test as the sample size was .50. To examine the impact of time (pre and post) and condition (WBVSS, SHAM) on the dependent variables, a 2-way repeated measures analysis of variance was used for the statistical analyses. Sphericity was checked using Mauchly's test, and the Greenhouse-Geisser's correction on degrees of freedom was applied when necessary. In cases where interaction between time and condition were detected, the simple effects were investigated, and Bonferroni's correction was used. In the absence of interaction, the main effects of the 2 factors (time and condition) on the dependent variables were investigated. Statistical significance waste at alpha $<0.05$.

\section{Results}

A statistically significant interaction effect was found on $\mathrm{K}_{\text {vert }}\left(\mathrm{F}_{(1,21)}=6.124, \mathrm{p}=.022, \mathrm{n}^{2}=\right.$ .226) between protocols (WBVSS, SHAM) and time (pre, post). The post hoc analysis of simple effects with Bonferroni correction revealed reduction for $\mathrm{K}_{\text {vert }}$ after the WBVSS protocol (WBVSS: $\mathrm{p}=.059$, SHAM: $\mathrm{p}=.981$ ).

Significant interaction effect was found on $K_{\operatorname{leg}}\left(F_{(1,21)}=16.040, p=.001, n 2=.433\right)$ between protocols and time. The post hoc analysis of simple effects with Bonferroni correction revealed a statistically significant decrease of leg stiffness after the WBVSS protocol (WBVSS: $\mathrm{p}=.017$, SHAM: $\mathrm{p}=.213$ ).

Statistical analysis indicated significant interaction effect between protocols and time for $\mathrm{F}_{\max }\left(\mathrm{F}_{(1,21)}=9.727, \mathrm{p}=.005, \mathrm{n} 2=.317\right)$. No main effect was found for protocols $(\mathrm{p}$ $>$.05). However, significant main effect was found for time $\left(\mathrm{F}_{(1,21)}=8.279, \mathrm{p}=.009, \mathrm{n} 2=\right.$ .283). The post hoc analysis of simple effects with Bonferroni correction revealed no significant increase for Fmax after the WBVSS protocol (WBVSS: $\mathrm{p}=.145$, SHAM: $\mathrm{p}=$ $.103)$.

Statistical analysis indicated significant interaction effect between protocols and time for $\Delta \mathrm{L}\left(\mathrm{F}_{(1,21)}=9.851, \mathrm{p}=.005, \mathrm{n} 2=.319\right)$. The post hoc analysis of simple effects with Bonferroni correction revealed significant increase for DL after the WBVSS protocol (WBVSS: $\mathrm{p}=.018$, SHAM: $\mathrm{p}=.637$ ).

Also, statistically significant interaction effect was found for contact time $\left(\mathrm{F}_{(1,21)}=\right.$ 12.466, $\mathrm{p}=.002, \mathrm{n} 2=.373$ ) between protocols and time. The post hoc analysis of simple effects with Bonferroni correction revealed statistically significant increase for contact time after the WBVSS protocol (WBVSS: $\mathrm{p}=.013$, SHAM: $\mathrm{p}=.345$ ).

Finally, a statistically significant interaction effect was found for flight time ( $F_{(1,21)}$ $=5.199, \mathrm{p}=.033, \mathrm{n} 2=.198)$ between protocols and time. No main effect was found for protocols $(\mathrm{p}>.05)$. However, a statistically significant main effect was found for time ( $\mathrm{F}$ $(1,21)=8.084, \mathrm{p}=.010, \mathrm{n} 2=.278)$. The post hoc analysis of simple effects with Bonferroni 
correction revealed statistically significant increase for flight time after the WBVSS protocol (WBVSS: $\mathrm{p}=.018$, SHAM: $\mathrm{p}=.637$ ).

\section{Discussion}

This is the first study that examined the acute effect of WBVSS on leg and vertical stiffness and the related spatiotemporal and kinetic characteristics during running. The results showed significant reduction for leg stiffness $(-3.24 \%)$, increased time contact $(0.96 \%)$, and $\triangle \mathrm{L}(2.44 \%)$ after the WBVSS protocol, whereas the rest the examined variables remained unchanged. The hypothesis that the SS combined with vibration (WBVSS) could affect positively the examined variables is rejected. The fact that the addition of vibration to a stretching routine maintains unchanged explosive strength of lower limbs (Dallas \& Kirialanis, 2013; Kinser et al, 2008) one would expect to have the same effect on leg and vertical stiffness. However, the present study revealed that WBVSS has a detrimental effect on leg stiffness and some kinetic and spatiotemporal variables as well. Our results opposed to those of Paradisis et al. (2019) who found that a protocol included 10 set of $30 \mathrm{~s}$ dynamic squatting exercises on the vibration platform significantly increased leg stiffness by $3.4 \%$, whereas time of contact and $\Delta \mathrm{L}$ revealed a no statistically significant reduction. Furthermore, our findings support partially previous data by Pappas et al. (2017) who examined the effect of SS and DS and found that neither SS nor DS altered leg or vertical stiffness, even though it has been proposed that both types of stretching reduce musculotendinous stiffness (MTS) (Herda et al, 2013). In addition, the present results reinforce findings by Hobara et al. (2011) who investigated the acute effects of stretching on stiffness and found no changes after 3-minute SS of the triceps surae. The reduced leg stiffness may be attributed to the decreased musculotendinous unit (MTU) stiffness which in turn is the result of increased compliance of the muscular portion of the MTU, rather than changes in tendon stiffness (Morse et al, 2008). The absence of changes in vertical stiffness reported in the present study may be due to the duration of applied stretch, which was selected taking into account the normal duration of the exercises practiced by athletes during warm-up or training.

The effects of the whole-body vibration such as increase in muscle temperature, better blood circulation, e.t.c. do not contribute to further performance improvement when combined with static stretching. It is speculated that SS combined with vibration have the same effect as SS alone. The results of the present study showed an imperceptible reduction in Fmax after WBVSS protocol. This finding is in accordance with other data that refer a performance reduction after SS application (Beckett et al, 2009; Gelen, 2010). It is possible in submaximal running intensities where lower levels of force are required, the more compliant tendon of quadriceps muscle benefit the production of quadriceps muscle in the run, because a smaller volume of activated muscle is involved in the required force production (Arampatzis et al, 2006). Further, as Wilson et al. (2010) stated SS have obliged to employ a larger number of motor units to perform the same mechanical work. Therefore, the maintenance of $F_{\max }$ after the application of SS is 
probably related to the fact that the activity following the stretching was not of maximum intensity. The increase in the mean value at contact time had a negative effect on flight time which decreased by $0.58 \%$, with a slight decrease in SR, while the SL remaining unchanged.

Several possible mechanisms are responsible for the diminishing effects of SS on force development. Previous data support that alterations in the mechanical (Weir et al, 2005) and morphological properties (Ryan et al. 2008) of the muscle tendon unit were responsible for the strength reduction after passive stretching which is suspected to further reduce the rate of force development that is being transferred to the bones (Power et al, 2004). Conclusively, the present study showed that a warm-up including SS combined with WBV did not produce significant effect on mean values of $\mathrm{K}_{\operatorname{leg}}$ and $\mathrm{K}_{\text {vert, }}$ and related kinetic and kinematic variables.

\section{Recommendations}

Further research is needed to examine the effect of WBV training combined with static stretching with athletes of other sports or/and to investigate the effect of intervention program on stiffness.

\section{Conflict of Interest}

No potential conflict of interest relevant to this article was reported.

\section{About the Authors}

Constantinos Ntallas is a teacher of Physical Education. Current Research interest: sport performance and strength training.

George Dallas is an associate at School of Physical Education and Sport Science, National and Kapodistrian University of Athens, Greece. Current research interest: the impact of selected physiological and anthropometrical variables in elite gymnastics performance, strength training.

\section{References}

Abercrombly A. F., Amonette W. E., Layne C. S., McFarlin B. K., Hinmasn M. R., and Paloski W. H. (2007). Vibration exposure and biodynamic responses during whole-body vibration training. Med Sci Sports Exe, 39, 1794-1800

Arampatzis, A., Schade, F., Walsh, M., and Bruggemann, G. P. (2001). Influence of leg stiffness and its effect on myodynamic jumping performance. J Electromyogr Kinesiol, 11, 355-364

Arampatzis, A., De Monte, G., Karamanidis, K., Morey-Klapsing, G., Stafilidis, S., and Bruggemann, G. P. (2006). Influence of the muscle-tendon unit's mechanical and morphological properties on running economy. J Experimental Biol, 209, 3345-3357 
Beckett, J. R. J., Schneiker, K. T., Wallman, K. E., Dawson, B. T., and Guelfi, K. J. (2009). Effects of static stretching on repeated sprint and change on direction performance. Med Sci Sports Exerc, 41(2), 444-450

Blickhan, R. (1989). The spring-mass model for running and hopping. J Biomech, 2, 12171227

Brughelli, M., and Cronin, J. (2008). Influence of running velocity on vertical, leg and joint stiffness: modelling and recommendations for future research. Sports Med, 38, 647657

Butler, R. J., Crowell, H. P., and Davis, I. M. (2003). Lower extremity stiffness: Implications for performance and injury. Clin Biomech, 18, 511-517

Cardinale, M., and Bosco, C. (2003). The use of vibration as an exercise intervention. Exerc Sport Sci Rev, 31, 3-7

Chelly, S., and Denis, C. (2002). Leg power and hopping stiffness: relationship with sprint running performance. Med Sci Sports Exerc, 33, 326-333

Cochrane, D. J., Loram, I., Stannard, S. R., and Rittweger, J. (2009). Changes in joint angles, muscle-tendon complex length, muscle contractile tissue displacement and modulation of EMG activity during acute whole-body vibration. Muscle Nerve, 40, 420-429

Colson, S. S., and Petit, P. D. (2013). Lower Limbs Power and Stiffness after Whole-Body Vibration. Int J Sports Med, 34, 318-323

Cronin, J. B., Oliver, M., and McNair, P. J. (2004). Muscle stiffness and injury effects of whole-body vibration. Phys Ther Sport, 5, 68-74

Dallas, G., Kaimakamis, V., Mellos, V., and Paradisis, G. (2012). Acute effect of wholebody vibration combined with stretching on bridge performance in artistic gymnasts. Biol Exe, 8(2), 47-57

Dallas, G., and Kirialanis, P. (2013). The effect of two different conditions of whole-body vibration on flexibility and jumping performance of artistic gymnasts. Sci Gymnastics J, 5(2), $67-77$

Dallas, G., Tsiganos, G., Tsolakis, Ch., Tsopani, D., Di Cagno, A., Smirniotou, A. (2014). The acute effect of different stretching methods on flexibility and jumping performance in competitive artistic gymnasts. J Sports Med Phys Fitness, 54 (6), 683690

Dalleau, G., Belli, A., Bourdin, M., and Lacour, J-R. (1998). The spring-mass model and the energy-cost of treadmill running. Eur J Applied Physiol Occup Physiol, 77, 257263

Derrick, T. R., Caldwell, G. E., and Hamill, J. (2000). Modeling the stiffness characteristics of the human body while running with various stride lengths. J Applied Biomech, $16,36-51$

Farley, C. T., Glasheen, J., and McMahon, T. A. (1993). Running springs: speed and animal seize. J Exp Biol, 185, 71-86

Gelen, E. (2010). Acute effects on different warm-up methods on sprint, slalom dribbling, and penalty kick performance in soccer players. J Strength Cond Res, 14(4), 960-956 
Herda, T. J., Ryan, E. D., Smith, A. E., Walter, A. A., Bemben, M. G., Stout, J.R., and Cramer, J.T. (2009). Acute effects of passive stretching vs vibration on the neuromuscular function of the plantar flexors. Scand J Med Sci Sports, 19, 703-713

Herda, T. J., Herda, N. D., Costa, P. B., Walter-Herda, A. A., Valdez, A. M., and Cramer, J. T. (2013). The effects of dynamic stretching on the passive properties of the muscle-tendon unit. J Sports Sci, 31, 479-487

Hobara H., Inoue, K., Gomi, K., Sakamoto, M., Muraoka, T., Iso, S., and Kanosue, K. (2010). Continuous change in spring-mass characteristics during a $400 \mathrm{~m}$ sprint. J Sci Med Sport, 13, 256-261

Hobara, H., Inoue, K., Kato, E., and Kanosue, K. (2011). Acute effects of static stretching on leg-spring behavior during hopping. Eur J Appl Physiol, 111, 2115-2121

Kinser, A. M., Ramsey, M. W., O’Bryant, H. S., Ayres, C. A., Sands, W. A., and Stone, M. H. (2008). Vibration and Stretching Effects on Flexibility and Explosive Strength in Young Gymnasts. Med Sci Sports Exerc, 40(1), 133-140

Kyröläinen, H., Pullinen, T., Candau, R., Avela, J., Huttunen, P., and Komi, P.V. (2000). Effects of marathon running on running economy and kinematics. Eur J Appl Physiol, 82, 297-304

McMahon, T. A., and Cheng, G. C. (1990). The mechanics of running: How does stiffness couple with speed? J Biomech, 23: 65-78

Morin, J. B., Dalleau, G., Kyröläinen, H., Jeannin, T., and Belli, A. (2005). A simple method for measuring stiffness during running. J Applied Biomech, 21, 167-180

Morse, C. I., Degens, H., Seynnes, O. R., Maganaris, C. N., and Jones, D. A. (2008). The acute effect of stretching on the passive stiffness of the human gastrocnemius muscle tendon unit. J Physiol, 586, 97-106

Nakamura, K., Kodama, T., and Mukaino, Y. (2014). Effects of active individual muscle stretching on muscle function. J Phys Ther Sci, 26, 341-344

Pappas, P., Paradisis, G. P., Exell, T. A., Smirniotou, A., Tsolakis, C., and Arampatzis, A. (2017). Acute effects of stretching on leg and vertical stiffness during treadmill running. J Strength Cond Res, 31(12), 3714-3724

Paradisis, G. P., and Cooke, C. B. (2001). Kinematic and postural characteristics of sprint running on sloping surfaces. J Sport Sci, 19, 149-159

Paradisis, G. P., Pappas, P. T., Theodorou, A. S., Zacharogiannis, E. G., Skordilis, E. K., and Smirniotou, A. S. (2014). Effects of static and dynamic stretching on sprint and jump performance in boys and girls J Strength Cond Res, 28, 154-160

Paradisis, G. P., Pappas, P., Dallas, G., Zacharogiannis, E., Rossi, J., Lapole, T. (2021). Acute effects of whole-body vibration warm-up on leg and vertical stiffness during running. J Strength Cond Res, 35(9), 2433-2438

Power, K., Behm, D., Cahill, F., Carroll, M., Young, W. (2004). An acute bout of static stretching: effects on force and jumping performance. Med Sci Sports Exerc, 36(8), 1389-1396 
Ronnestad, B. R. (2004). Comparing the performance-enhancing effects of squats on a vibration platform with conventional squats in recreationally resistance-trained men. J Strength Cond Res, 18, 839-845

Ryan, E. D., Beck, T. W., Herda, T. J., Hull, H. R., Hartman, M. J., Costa, P. B., Defreitas, J. M., Stout, J. R., and Cramer, J. T. (2008). The time course of musculotendinous stiffness responses following different durations of passive stretching. J Orthop Sports Phys Ther, 38(10), 632-639

Sands, W. A., McNeal, J. R., Stone, M. H., Haff, G. G., and Kinser, A. M. (2008). Effect of vibration on forward split flexibility and pain perception in young male gymnasts. Int J Sport Physiol Perform, 3, 469-481

Siu, P. M., Tam, B. T., Chow, D. H., Go, Y. P., Huang, Y. P., Zheng, Y. P., and Wong, S. H. (2010). Immediate effects of 2 different whole-body vibration frequencies on muscle peak torque and stiffness. rch Phys Med Rehabil, 91, 1608-1615

Stone, M. H., Ramsey, M. W., Kinser, A. M., O'Bryant, H. S., Ayres, C., Sands, W. A. (2006). Stretching: acute and chronic? The potential consequences. Strength Cond J, $28,66-74$

Wakeling, J. M., Nigg, B. M., and Rozitis, A. I. Muscle activity damps the soft tissue resonance that occurs in response to pulsed and continuous vibrations. J Appl Physiol, 93, 1093-1103

Weir, D. E., Tingley, J., Elder, G. C. (2005). Acute passive stretching alters the mechanical properties of human plantar flexors and the optimal angle for maximal voluntary contraction. Eur J Appl Physiol, 93(5-6), 614-623

Wilson, J. M., Hornbuckle, L. M., Kim, J. S., Ugrinowitsch, C., Lee, S. R., Zourdos, M. C., Sommer, B., and Panton, L. B. (2010). Effects of static stretching on energy cost and running endurance performance. J Strength Cond Res, 24, 2274-2279

Yapicioglu, B., Colakoglu, M., Colakoglu, Z., Gulluoglu, H., Bademkiran, F., and Ozkaya, O. (2013). Effects of a Dynamic Warm-up, Static Stretching or Static Stretching with Tendon Vibration on Vertical Jump Performance and EMG Responses. J Hum Kin, 39, 49-57 


\section{Appendix}

Table 1: Mean values, standard deviation and percentage difference for stiffness, kinetic and kinematic variables between pre and post measurements for static stretching combined with vibration and control

\begin{tabular}{|l|c|c|c|c|c|}
\hline \multicolumn{7}{|l|}{ Kleg (kN . m-1) } & Kvert (kN . m-1) & Fmax (kN) & $\Delta$ L (cm) & $\Delta y(\mathbf{c m})$ \\
\hline WBVSS & $7.712 \pm 1.612$ & $27.276 \pm 3.727$ & $1.395 \pm 0.198$ & $0.183 \pm 0.020$ & $0.051 \pm 0.005$ \\
\hline Pre & $7.462 \pm 1.607^{*}$ & $26.779 \pm 3.681$ & $1.382 \pm 0.188$ & $0.188 \pm 0.020^{*}$ & $0.051 \pm 0.005$ \\
\hline Post & -3.24 & -1.82 & -0.89 & 2.44 & 0.97 \\
\hline$\Delta \%$ & $7.605 \pm 1.588$ & $26.747 \pm 3.610$ & $1.400 \pm 0.192$ & $0.186 \pm 0.019$ & $0.052 \pm 0.005$ \\
\hline SHAM & $26.742 \pm 3.712$ & $1.415 \pm 0.190$ & $0.186 \pm 0.020$ & $0.053 \pm 0.005$ \\
\hline Pre & $7.735 \pm 1.702$ & -0.01 & 1.08 & -0.36 & 0.85 \\
\hline Post & 1.71 & &
\end{tabular}

*Significantly different from pre $(\mathrm{p}<0.05)$ as determined by repeated-measures analysis of variance and post hoc tests. Kleg: leg stiffness, Kvert: vertical stiffness, Fmax: maximal ground reaction force, $\Delta \mathrm{L}$ : change in leg length, $\Delta y$ : vertical displacement of the center of mass, WBVSS: vibration plus static stretching, SHAM: control.

Table 2: Mean values, standard deviation and percentage difference for temporal and spatiotemporal variables between pre and post measurements for static stretching combined with vibration and control

\begin{tabular}{|l|c|c|c|c|}
\hline & Tc (ms) & Tf (ms) & SR (Hz) & SL (m) \\
\hline WBVSS & \multicolumn{5}{|l|}{} \\
\hline Pre & $0.208 \pm 0.014$ & $0.113 \pm 0.016$ & $3.11 \pm 0.15$ & $1.43 \pm 0.07$ \\
\hline Post & $0.210 \pm 0.015^{*}$ & $0.112 \pm 0.017$ & $3.09 \pm 0.14$ & $1.43 \pm 0.06$ \\
\hline$\Delta \%$ & 0.96 & -0.88 & 0.58 & -0.48 \\
\hline SHAM & $0.209 \pm 0.014$ & $0.115 \pm 0.016$ & $3.07 \pm 0.14$ & $1.44 \pm 0.06$ \\
\hline Pre & $0.208 \pm 0.015$ & $0.118 \pm 0.017$ & $3.06 \pm 0.14$ & $1.45 \pm 0.06$ \\
\hline Post & 1.96 & 2.61 & 0.58 & -0.61 \\
\hline$\Delta \%$ &
\end{tabular}

*Significantly different from pre $(\mathrm{p}<0.05)$ as determined by repeated-measures analysis of variance and post hoc tests. Tc: contact time, Tf: flight time, SR: step rate, SL: step length, WBVSS: vibration plus static stretching, SHAM: control. 
Creative Commons licensing terms

Authors will retain the copyright of their published articles agreeing that a Creative Commons Attribution 4.0 International License (CC BY 4.0) terms will be applied to their work. Under the terms of this license, no permission is required from the author(s) or publisher for members of the community to copy, distribute, transmit or adapt the article content, providing a proper, prominent and unambiguous attribution to the authors in a manner that makes clear that the materials are being reused under permission of a Creative Commons License. Views, opinions and conclusions expressed in this research article are views, opinions and conclusions of the author(s). Open Access Publishing Group and European Journal of Physical Education and Sport Science shall not be responsible or answerable for any loss, damage or liability caused in relation to/arising out of conflict of interests, copyright violations and inappropriate or inaccurate use of any kind content related or integrated on the research work. All the published works are meeting the Open Access Publishing requirements and can be freely accessed, shared, modified, distributed and used in educational, commercial and non-commercial purposes under a Creative Commons attribution 4.0 International License (CC BY 4.0). 